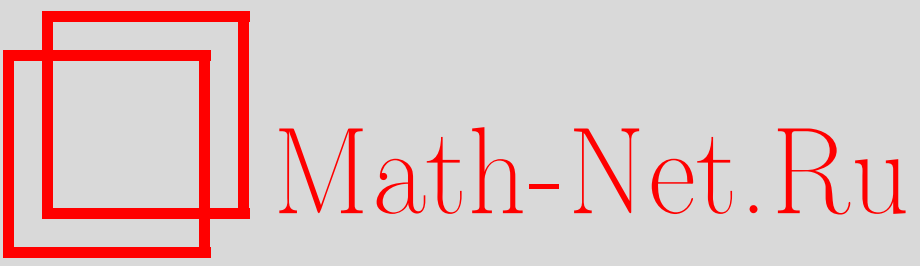

А. Б. Пичкур, Описание класса подстановок, представимых в виде произведения двух подстановок с фиксированным числом мобильных точек, Матем. вопр. криптогр., 2012, том 3, выпуск 2, 79-95

DOI: https://doi.org/10.4213/mvk55

Использование Общероссийского математического портала Math-Net.Ru подразумевает, что вы прочитали и согласны с пользовательским соглашением

http: //www . mathnet.ru/rus/agreement

Параметры загрузки:

IP : 54.197 .217 .227

26 апреля 2023 г., 11:20:50 


\title{
Описание класса подстановок, представимых в виде произведения двух подстановок с фиксированным числом мобильных точек
}

\author{
А. Б. Пичкур \\ ООО «Центр сертификационных исследований», Москва
}

Получено 20.V.2011

В работе полностью описано строение класса подстановок степени $N$, представимых в виде произведения двух подстановок с $q$ мобильными и $N-q$ неподвижными точками, $4 \leq q \leq \frac{N}{2}$.

Ключевые слова: симметрическая группа, мультипликативные представления, неподвижные точки

Description of the set of permutations represented as a product of two permutations with fixed number of mobile points

\section{A. B. Pichkur}

LLC "Certification Research Center", Moscow

Abstract. We describe completely the structure of the set of permutations representable by the product of two permutations with $q$ mobile and $N-q$ fixed points, $4 \leq q \leq \frac{N}{2}$.

Key words: symmetric group, multiplicative representations, fixed points

Citation: Mathematical Aspects of Cryptography, 2012, vol. 3, no. 2, pp. $79-95$ (Russian). 
Пусть подстановка $G \in S_{N}, \Gamma(G) \subseteq\{1, \ldots, N\}$ - множество мобильных точек подстановки $G, 2 \leq q \leq N-$ натуральное число. Обозначим через

$$
\Gamma_{N}(q)=\left\{G \in S_{N}|| \Gamma(G) \mid=q\right\}
$$

множество всех подстановок степени $N$, имеющих ровно $q$ мобильных (и, значит, $N-q$ неподвижных) точек.

В данной работе описано множество всех подстановок из $\Gamma_{N}(q) \cdot \Gamma_{N}(q)$, т. е. всех подстановок, представимых в виде произведения двух подстановок c $q$ мобильными точками. Эта задача связана с приложениями в криптографии.

В научной литературе рассматривается схожая задача описания множества подстановок, принадлежащих произведению двух или более классов сопряженных элементов из $S_{N}$ (или из $A_{N}$ ). При этом основное внимание уделяется вычислению двух параметров: числу покрытия (covering number) и расширенному числу покрытия (extended covering number) $S_{N}$ (или $A_{N}$ ).

Под числом покрытия группы $G$ понимается наименьшее натуральное число $k$, удовлетворяющее условию: для любого нетривиального класса $C$ сопряженных элементов группы $G$ выполняется равенство $C^{k}=G$.

Под расширенным числом покрытия группы $G$ понимается наименьшее натуральное число $k$, удовлетворяющее условию: для любых нетривиальных классов $C_{1}, \ldots, C_{k}$ сопряженных элементов группы $G$ выполняется равенство $C_{1} \cdot \ldots \cdot C_{k}=G$.

Также для фиксированного класса сопряженных элементов $C$ изучалось значение экспоненты $C$, т. е. наименьшего натурального $k$ со свойством $C^{k}=G$.

В списке литературы приведен неполный перечень публикаций по указанной теме. Ряд важных результатов можно найти в монографии [10].

Так как $\Gamma_{N}(q)$ является объединением классов сопряженных элементов из $S_{N}$, то для решения поставленной задачи можно использовать некоторые из известных результатов. В частности, в [1] доказана следующая теорема.

Теорема 1. Пусть $G \in A_{N}, c(G)$ - число неединичных цииклов в $G$, $q>0 u \frac{c(G)+|\Gamma(G)|}{2} \leq q \leq N$. Тогда существуют такие q-циикль $P_{1}, P_{2}$, что $G=P_{1} \cdot P_{2}$.

Следующая теорема, справедливая и для нечетных подстановок, была опубликована автором в 1990 г. в ведомственном издании. 
Tеорема 2. Пусть $N \geq 5,4 \leq q \leq N, G \in S_{N}$. Если $|\Gamma(G)| \leq\left[\frac{4}{3} q\right]+1$, то существуют $q$-цикл $P_{1}$ и подстановка $P_{2} \in \Gamma_{N}(q)$, для которых выполняется равенство $G=P_{1} \cdot P_{2}$.

ЗАМЕЧАНИЕ. Из теоремы 2 , в частности, следует, что при $N \geq 5$, $4 \leq q \leq N \quad$ в $\quad \Gamma_{N}(q) \cdot \Gamma_{N}(q) \quad$ содержатся все множества $\Gamma_{N}(r), \quad$ где $2 \leq r \leq\left[\frac{4}{3} q\right]+1$. Однако этот результат описывает строение множества $\Gamma_{N}(q) \cdot \Gamma_{N}(q)$ лишь частично.

Ниже строение множества $\Gamma_{N}(q) \cdot \Gamma_{N}(q)$ будет изучено полностью. Сначала докажем важное для дальнейших рассуждений вспомогательное утверждение.

Утверждение 3. Если $N \geq 6,2 \leq q_{1}<q_{2} \leq \frac{N}{2}$, то имеет место включение $\Gamma_{N}\left(q_{1}\right) \cdot \Gamma_{N}\left(q_{1}\right) \subseteq \Gamma_{N}\left(q_{2}\right) \cdot \Gamma_{N}\left(q_{2}\right)$.

Доказательство. Пусть $H \in \Gamma_{N}\left(q_{1}\right) \cdot \Gamma_{N}\left(q_{1}\right)$, т. е. для некоторых $H_{1}, H_{2} \in \Gamma_{N}\left(q_{1}\right) \quad$ имеет место равенство $H=H_{1} \cdot H_{2}$. Так как $\left|\Gamma\left(H_{1}\right) \cup \Gamma\left(H_{2}\right)\right| \leq 2 \cdot q_{1}$, то

$$
\left|\Omega \backslash\left(\Gamma\left(H_{1}\right) \cup \Gamma\left(H_{2}\right)\right)\right|=N-\left|\Gamma\left(H_{1}\right) \cup \Gamma\left(H_{2}\right)\right| \geq N-2 q_{1} \geq 2 q_{2}-2 q_{1} \geq 2\left(q_{2}-q_{1}\right) .
$$

1. Если $q_{2}-q_{1} \geq 2$, то в множестве $\Omega \backslash\left(\Gamma\left(H_{1}\right) \cup \Gamma\left(H_{2}\right)\right)$ можно выбрать $t=q_{2}-q_{1}$ элементов $\left\{\alpha_{1}, . ., \alpha_{t}\right\}$ и получить равенство

$$
H=\left(H_{1} \cdot\left(\alpha_{1}, . ., \alpha_{t}\right)\right) \cdot\left(H_{2} \cdot\left(\alpha_{t}, . ., \alpha_{2}, \alpha_{1}\right)\right) .
$$

Действительно, так как

$$
\Gamma\left(\left(\alpha_{1}, . ., \alpha_{t}\right)\right) \cap \Gamma\left(H_{1}\right)=\varnothing, \quad \Gamma\left(\left(\alpha_{1}, . ., \alpha_{t}\right)\right) \cap \Gamma\left(H_{2}\right)=\varnothing,
$$

то

$$
\begin{gathered}
\left(H_{1} \cdot\left(\alpha_{1}, . ., \alpha_{t}\right)\right) \cdot\left(H_{2} \cdot\left(\alpha_{t}, . ., \alpha_{2}, \alpha_{1}\right)\right)= \\
=H_{1} \cdot H_{2} \cdot\left(\alpha_{1}, . ., \alpha_{t}\right) \cdot\left(\alpha_{t}, . ., \alpha_{2}, \alpha_{1}\right)=H_{1} \cdot H_{2}=H, \\
\left|\Gamma\left(H_{1} \cdot\left(\alpha_{1}, . ., \alpha_{t}\right)\right)\right|=\left|\Gamma\left(H_{1}\right)\right|+t=q_{1}+t=q_{2}, \\
\left|\Gamma\left(H_{2} \cdot\left(\alpha_{t}, . ., \alpha_{1}\right)\right)\right|=\left|\Gamma\left(H_{2}\right)\right|+t=q_{1}+t=q_{2} .
\end{gathered}
$$

Итак, доказано, что $H \in \Gamma_{N}\left(q_{2}\right) \cdot \Gamma_{N}\left(q_{2}\right)$. 
2. Пусть теперь $q_{2}-q_{1}=1$.

2a. Если при этом $\Gamma\left(H_{1}\right) \neq \Gamma\left(H_{2}\right)$, то существуют $\alpha_{1} \in \Gamma\left(H_{1}\right) \backslash \Gamma\left(H_{2}\right)$ и $\beta_{1} \in \Gamma\left(H_{2}\right) \backslash \Gamma\left(H_{1}\right)$. Рассмотрим соответствующие циклы из цикловой записи подстановок $H_{1}, H_{2}$ :

$$
H_{1}=\left(\alpha_{1}, \ldots, \alpha_{r}\right) \cdot H_{1}^{\prime}, \quad H_{2}=\left(\beta_{1}, \ldots, \beta_{s}\right) \cdot H_{2}^{\prime} .
$$

Нетрудно убедиться в справедливости равенства

$$
\begin{gathered}
\left(\left(\alpha_{1}, \ldots, \alpha_{r}, \beta_{1}\right) \cdot H_{1}{ }^{\prime}\right)\left(\left(\beta_{1}, \alpha_{1}, \beta_{2}, \ldots, \beta_{s}\right) \cdot H_{2}{ }^{\prime}\right)= \\
=\left(\left(\alpha_{1}, \ldots, \alpha_{r}\right) \cdot H_{1}{ }^{\prime}\right)\left(\left(\beta_{1}, \ldots, \beta_{s}\right) \cdot H_{2}{ }^{\prime}\right)=H_{1} \cdot H_{2}=H .
\end{gathered}
$$

Так как

$$
\begin{gathered}
\left|\Gamma\left(\left(\alpha_{1}, \ldots, \alpha_{r}, \beta_{1}\right) \cdot H_{1}{ }^{\prime}\right)\right|=q_{1}+1=q_{2}, \\
\left|\Gamma\left(\left(\beta_{1}, \alpha_{1}, \beta_{2}, \ldots, \beta_{s}\right) \cdot H_{2}{ }^{\prime}\right)\right|=q_{1}+1=q_{2},
\end{gathered}
$$

то полученное равенство означает, что $H \in \Gamma_{N}\left(q_{2}\right) \cdot \Gamma_{N}\left(q_{2}\right)$.

2б. Рассмотрим оставшийся случай $\Gamma\left(H_{1}\right)=\Gamma\left(H_{2}\right)$. В этом случае $\Gamma(H) \subseteq \Gamma\left(H_{1}\right)$ и, значит, $|\Gamma(H)| \leq q_{1}$. Тогда

$$
\left|\Omega \backslash\left(\Gamma\left(H_{1}\right) \cup \Gamma\left(H_{2}\right)\right)\right|=N-\left|\Gamma\left(H_{1}\right)\right|=N-q_{1}>N-\frac{N}{2}=\frac{N}{2} \geq 3 .
$$

Полученное условие означает, что можно выбрать точку $\delta \in \Omega \backslash \Gamma\left(H_{1}\right)$.

Если при этом $q_{1}=2$, то сразу получаем, что $H_{1}=H_{2}$ - транспозиция. Тогда $H=H_{1} \cdot H_{2}=E$ - тождественная подстановка. В этом случае включение $H \in \Gamma_{N}\left(q_{2}\right) \cdot \Gamma_{N}\left(q_{2}\right)$ очевидно.

Пусть теперь $q_{1} \geq 3$. Рассмотрим два возможных случая.

Сначала предположим, что $\Gamma(H) \neq \Gamma\left(H_{1}\right)$. Это означает, что существует $\alpha \in \Gamma\left(H_{1}\right)$ со свойством $H(\alpha)=\alpha$. Отсюда следует, что для некоторого $\beta \neq \alpha, \beta \in \Gamma\left(H_{1}\right)$ выполняются равенства $H_{1}(\alpha)=\beta, H_{2}(\beta)=\alpha$. Тогда выберем $\delta \in \Omega \backslash \Gamma\left(H_{1}\right)$ и положим

$$
H_{1}^{\prime}(x)=\left\{\begin{array}{lrr}
\delta, & x=\alpha, \\
\beta, & x=\delta, \\
H_{1}(x), & x \in \Gamma\left(H_{1}\right) \backslash\{\alpha\},
\end{array} \quad H_{2}^{\prime}(x)=\left\{\begin{array}{lr}
\delta, & x=\beta, \\
\alpha, & x=\delta, \\
H_{2}(x), & x \in \Gamma\left(H_{1}\right) \backslash\{\beta\} .
\end{array}\right.\right.
$$


Непосредственно проверяется, что $H=H_{1}^{\prime} \cdot H_{2}^{\prime}$. Так как при этом

$$
\left|\Gamma\left(H_{1}{ }^{\prime}\right)\right|=q_{1}+1=q_{2}, \quad\left|\Gamma\left(H_{2}{ }^{\prime}\right)\right|=q_{1}+1=q_{2},
$$

то полученное равенство означает, что $H \in \Gamma_{N}\left(q_{2}\right) \cdot \Gamma_{N}\left(q_{2}\right)$.

Наконец, пусть $\Gamma(H)=\Gamma\left(H_{1}\right)$. Это означает, что для любого $\alpha \in \Gamma\left(H_{1}\right)$ $H_{1}(\alpha)=\beta, \quad \beta \neq \alpha \quad$ и $H_{2}(\beta)=\gamma, \quad \gamma \neq \alpha, \gamma \neq \beta$. Зафиксируем некоторый $\alpha \in \Gamma\left(H_{1}\right)$, выберем $\delta \in \Omega \backslash \Gamma\left(H_{1}\right)$ и положим

$$
H_{1}^{\prime}(x)=\left\{\begin{array}{lr}
\delta, & x=\alpha, \\
\beta, & x=\delta, \\
H_{1}(x), & x \in \Gamma\left(H_{1}\right) \backslash\{\alpha\},
\end{array} \quad H_{2}^{\prime}(x)=\left\{\begin{array}{lr}
\delta, & x=\beta, \\
\gamma, & x=\delta, \\
H_{2}(x), & x \in \Gamma\left(H_{1}\right) \backslash\{\beta\} .
\end{array}\right.\right.
$$

Непосредственно проверяется, что $H=H_{1}^{\prime} \cdot H_{2}^{\prime}$. Так как при этом

$$
\left|\Gamma\left(H_{1}^{\prime}\right)\right|=q_{1}+1=q_{2}, \quad\left|\Gamma\left(H_{2}{ }^{\prime}\right)\right|=q_{1}+1=q_{2},
$$

то полученное равенство означает, что $H \in \Gamma_{N}\left(q_{2}\right) \cdot \Gamma_{N}\left(q_{2}\right)$.

Утверждение доказано полностью.

Сформулируем основной результат работы.

Теорема 4. Пусть $N \geq 8,4 \leq q \leq \frac{N}{2}, G \in S_{N}$. Если $|\Gamma(G)| \leq 2 q-2$, то существуют подстановки $H_{1}, H_{2} \in \Gamma_{N}(q)$, для которых выполняется равенство $G=H_{1} \cdot H_{2}$.

Прежде чем приступать к доказательству этой теоремы, проверим ее выполнение для небольших значений $q \in\{4,5,6\}$.

Сначала отметим один очевидный факт: если $H \in \Gamma_{N}(q) \cdot \Gamma_{N}(q)$, то любая подстановка, сопряженная с $H$, также лежит в $\Gamma_{N}(q) \cdot \Gamma_{N}(q)$. Действительно, если $H=H_{1} \cdot H_{2}, H_{1}, H_{2} \in \Gamma_{N}(q)$ и $G=X^{-1} \cdot H \cdot X$, то

$$
G=\left(X^{-1} H_{1} X\right) \cdot\left(X^{-1} H_{2} X\right), \quad X^{-1} H_{1} X \in \Gamma_{N}(q), \quad X^{-1} H_{2} X \in \Gamma_{N}(q) .
$$

Значит, множество $\Gamma_{N}(q) \cdot \Gamma_{N}(q)$ является объединением некоторых классов сопряженных в группе $S_{N}$ подстановок.

1. Пусть $q=4$. Согласно теореме 4 должно выполняться включение

$$
\Gamma_{N}(2) \cup \Gamma_{N}(3) \cup \Gamma_{N}(4) \cup \Gamma_{N}(5) \cup \Gamma_{N}(6) \subseteq \Gamma_{N}(4) \cdot \Gamma_{N}(4) .
$$

С учетом сделанного выше замечания для проверки этого свойства необходимо выписать все возможные цикловые структуры подстановок из 
$\Gamma_{N}(2) \cup \Gamma_{N}(3) \cup \Gamma_{N}(4) \cup \Gamma_{N}(5) \cup \Gamma_{N}(6)$ и в каждом случае указать подстановки из $\Gamma_{N}(4)$, произведение которых дает подстановку с нужной цикловой структурой. Выпишем результаты расчетов в виде таблицы.

\begin{tabular}{|l|l|l|}
\hline & $\begin{array}{l}\text { Цикловые } \\
\text { структуры }\end{array}$ & \multicolumn{1}{|c|}{$\begin{array}{c}\text { Представление в виде произведения } \\
\text { двух подстановок из } \Gamma_{N}(4)\end{array}$} \\
\hline$\Gamma_{N}(2)$ & {$\left[2^{1}\right]$} & $(1,2,3,4) \cdot(1,2)(3,4)=(2,4)$ \\
\hline$\Gamma_{N}(3)$ & {$\left[3^{1}\right]$} & $(1,2,3,4) \cdot(3,2,1,5)=(3,4,5)$ \\
\hline \multirow{3}{*}{$\Gamma_{N}(4)$} & {$\left[2^{2}\right]$} & $(1,2,3,4) \cdot(1,2,3,4)=(1,3)(2,4)$ \\
\cline { 2 - 3 } & {$\left[4^{1}\right]$} & $(1,2,3,4) \cdot(1,3)(2,4)=(1,4,3,2)$ \\
\hline \multirow{3}{*}{$\Gamma_{N}(5)$} & {$\left[2^{1}, 3^{1}\right]$} & $(1,2,3,4) \cdot(1,3)(2,5)=(1,5,2)(3,4)$ \\
\cline { 2 - 3 } & {$\left[5^{1}\right]$} & $(1,2,3,4) \cdot(1,2,3,5)=(1,3,4,2,5)$ \\
\hline \multirow{5}{*}{$\Gamma_{N}(6)$} & {$\left[2^{3}\right]$} & $(1,2,3,4) \cdot(1,3)(5,6)=(1,2)(3,4)(5,6)$ \\
\cline { 2 - 3 } & {$\left[2^{1}, 4^{1}\right]$} & $(1,2,3,4) \cdot(3,5,4,6)=(1,2,5,4)(3,6)$ \\
\cline { 2 - 3 } & {$\left[3^{2}\right]$} & $(1,2,3,4) \cdot(3,4,5,6)=(1,2,4)(3,5,6)$ \\
\cline { 2 - 3 } & {$\left[6^{1}\right]$} & $(1,2,3,4) \cdot(1,6)(3,5)=(1,2,5,3,4,6)$ \\
\hline
\end{tabular}

2. Пусть $q=5$. В силу утверждения 3

$\Gamma_{N}(2) \cup \Gamma_{N}(3) \cup \Gamma_{N}(4) \cup \Gamma_{N}(5) \bigcup \Gamma_{N}(6) \subseteq \Gamma_{N}(4) \cdot \Gamma_{N}(4) \subseteq \Gamma_{N}(5) \cdot \Gamma_{N}(5)$.

Поэтому осталось только проверить включение $\Gamma_{N}(7) \cup \Gamma_{N}(8) \subseteq$ $\subseteq \Gamma_{N}(5) \cdot \Gamma_{N}(5)$. Снова выпишем результаты расчетов в виде таблицы.

\begin{tabular}{|l|l|l|}
\hline & \multicolumn{1}{|c|}{$\begin{array}{c}\text { Цикловые } \\
\text { структуры }\end{array}$} & \multicolumn{1}{c|}{$\begin{array}{c}\text { Представление в виде произведения } \\
\text { двух подстановок из } \Gamma_{N}(5)\end{array}$} \\
\hline \multirow{4}{*}{$\Gamma_{N}(7)$} & {$\left[7^{1}\right]$} & $(1,2,3,4,5) \cdot(1,2,3,6,7)=(1,3,4,5,2,6,7)$ \\
\cline { 2 - 3 } & {$\left[2^{1}, 5^{1}\right]$} & $(1,2,3,4,5) \cdot(2,6)(4,5,7)=(1,6,2,3,5)(4,7)$ \\
\cline { 2 - 3 } & {$\left[2^{2}, 3^{1}\right]$} & $(1,2)(3,4,5) \cdot(3,4,5)(6,7)=(1,2)(3,5,4)(6,7)$ \\
\cline { 2 - 3 } & {$\left[3^{1}, 4^{1}\right]$} & $(1,2,3,4,5) \cdot(2,5)(1,6,7)=(1,5,6,7)(2,3,4)$ \\
\hline
\end{tabular}


Окончание таблицы

\begin{tabular}{|l|l|l|}
\hline & \multicolumn{1}{|c|}{$\begin{array}{c}\text { Цикловые } \\
\text { структуры }\end{array}$} & \multicolumn{1}{c|}{$\begin{array}{c}\text { Представление в виде произведения } \\
\text { двух подстановок из } \Gamma_{N}(5)\end{array}$} \\
\hline & {$\left[8^{1}\right]$} & $(1,2,3,4,5) \cdot(2,6,7)(5,8)=(1,6,7,2,3,4,8,5)$ \\
\cline { 2 - 3 } & {$\left[2^{1}, 6^{1}\right]$} & $(1,2,3,4,5) \cdot(2,6,1,7,8)=(1,6)(2,3,4,5,7,8)$ \\
\hline$\Gamma_{N}(8)$ & {$\left[2^{2}, 4^{1}\right]$} & $(1,2,3,4,5) \cdot(1,2,6)(7,8)=(1,6)(2,3,4,5)(7,8)$ \\
\cline { 2 - 3 } & {$\left[2^{4}\right]$} & $(1,2)(3,4,5) \cdot(3,4,6)(7,8)=(1,2)(3,6)(4,5)(7,8)$ \\
\hline$\left[2^{1}, 3^{2}\right]$ & $(1,2,3,4,5) \cdot(3,5)(6,7,8)=(1,2,5)(3,4)(6,7,8)$ \\
\hline$\left[3^{1}, 5^{1}\right]$ & $(1,2,3,4,5) \cdot(1,3,6,7,8)=(1,2,6,7,8)(3,4,5)$ \\
\hline$\left[4^{2}\right]$ & $(1,2,3,4,5) \cdot(1,2,6,7,8)=(1,6,7,8)(2,3,4,5)$ \\
\hline
\end{tabular}

3. Пусть теперь $q=6$. В силу утверждения 3

$$
\Gamma_{N}(2) \cup \ldots \cup \Gamma_{N}(8) \subseteq \Gamma_{N}(5) \cdot \Gamma_{N}(5) \subseteq \Gamma_{N}(6) \cdot \Gamma_{N}(6) .
$$

Поэтому осталось проверить включение $\Gamma_{N}(9) \cup \Gamma_{N}(10) \subseteq \Gamma_{N}(6) \cdot \Gamma_{N}(6)$. Снова выпишем результаты расчетов в виде таблицы.

\begin{tabular}{|l|l|l|}
\hline & $\begin{array}{c}\text { Цикловые } \\
\text { структуры }\end{array}$ & \multicolumn{1}{|c|}{$\begin{array}{c}\text { Представление в виде произведения } \\
\text { двух подстановок из } \Gamma_{N}(6)\end{array}$} \\
\hline$\left[\begin{array}{l}{\left[9^{1}\right]} \\
\Gamma_{N}(9)\end{array}\right.$ & $(1,2,3,4,5,6) \cdot(1,2,3,7,8,9)=(1,3,4,5,6,2,7,8,9)$ \\
\hline$\left[2^{1}, 7^{1}\right]$ & $(1,2,3,4,5,6) \cdot(1,2,3,7)(8,9)=(1,3,4,5,6,2,7)(8,9)$ \\
\hline$\left[2^{2}, 5^{1}\right]$ & $(1,2,3,4)(5,6) \cdot(1,3)(5,7)(8,9)=(1,2)(3,4)(5,6,7)(8,9)$ \\
\hline$\left[2^{3}, 3^{1}\right]$ & $(1,2,3,4)(5,6) \cdot(1,2,3,7)(8,9)=(1,3,4,2,7)(5,6)(8,9)$ \\
\hline$\left[2^{1}, 3^{1}, 4^{1}\right]$ & $(1,2,3,4)(5,6) \cdot(1,8,2,9)(5,7)=(1,9)(5,6,7)(2,3,4,8)$ \\
\hline$\left[3^{1}, 6^{1}\right]$ & $(1,2,3,4)(5,6) \cdot(1,8)(5,7)(2,9)=(1,9,2,3,4,8)(5,6,7)$ \\
\hline$\left[3^{3}\right]$ & $(1,2,3)(4,5,6) \cdot(4,5,6)(7,8,9)=(1,2,3)(4,6,5)(7,8,9)$ \\
\hline$\left[4^{1}, 5^{1}\right]$ & $(1,2,3,4,5,6) \cdot(1,7,8,9)(2,6)=(1,6,7,8,9)(2,3,4,5)$ \\
\hline
\end{tabular}


Окончание таблицы

\begin{tabular}{|l|l|l|}
\hline & $\begin{array}{l}\text { Цикловые } \\
\text { структуры }\end{array}$ & \multicolumn{1}{|c|}{$\begin{array}{c}\text { Представление в виде произведения } \\
\text { двух подстановок из } \Gamma_{N}(6)\end{array}$} \\
\hline$\left[10^{1}\right]$ & $(1,2,3,4,5,6) \cdot(1,7,8)(2,9,10)=(1,9,10,2,3,4,5,6,7,8)$ \\
\cline { 2 - 3 } & {$\left[\begin{array}{l}{\left[2^{1}, 8^{1}\right]} \\
\right.$\cline { 2 - 3 }\end{array}} & $(1,2,3,4,5,6) \cdot(1,8,9,10,2,7)=(1,7)(2,3,4,5,6,8,9,10)$ \\
\hline$\left[2^{2}, 6^{1}\right]$ & $(1,2,3,4)(5,6) \cdot(1,7)(2,8)(9,10)=(1,8,2,3,4,7)(5,6)(9,10)$ \\
\hline$\left[2^{3}, 4^{1}\right]$ & $(1,2,3,4)(5,6) \cdot(1,7,2,8)(9,10)=(1,8)(2,3,4,7)(5,6)(9,10)$ \\
\hline$\left[2^{5}\right]$ & $(1,2,3,4)(5,6) \cdot(1,3)(7,8)(9,10)=(1,2)(3,4)(5,6)(7,8)(9,10)$ \\
\hline$\left[2^{2}, 3^{2}\right]$ & $(1,2,3)(4,5,6) \cdot(4,5,7)(8,9,10)=(1,2,3)(4,7)(5,6)(8,9,10)$ \\
\hline$\left[2^{1}, 3^{1}, 5^{1}\right]$ & $(1,2,3,4)(5,6) \cdot(5,7)(2,8)(9,10)=(1,8,2,3,4)(5,6,7)(9,10)$ \\
\hline$\left[2^{1}, 4^{2}\right]$ & $(1,2,3,4,5,6) \cdot(1,5)(7,8,9,10)=(1,2,3,4)(5,6)(7,8,9,10)$ \\
\hline$\left[3^{1}, 7^{1}\right]$ & $(1,2,3,4)(5,6) \cdot(1,8,9,10)(5,7)=(1,2,3,4,8,9,10)(5,6,7)$ \\
\hline$\left[3^{2}, 4^{1}\right]$ & $(1,2,3,4,5,6) \cdot(1,4)(7,8,9,10)=(1,2,3)(4,5,6)(7,8,9,10)$ \\
\hline$\left[4^{1}, 6^{1}\right]$ & $(1,2,3,4)(5,6) \cdot(1,9,10)(5,7,8)=(1,2,3,4,9,10)(5,6,7,8)$ \\
\hline$\left[5^{2}\right]$ & $(1,2,3,4,5,6) \cdot(1,2,7,8,9,10)=(1,7,8,9,10)(2,3,4,5,6)$ \\
\hline
\end{tabular}

\section{Доказательство теоремы 4.}

1. Выше было показано, что теорема 4 верна при $q \in\{4,5,6\}$. Далее доказательство теоремы проводится индукцией по $q$.

Пусть $q>6$. Если $G \in S_{N}$ и $|\Gamma(G)| \leq 2 q-4$, то по предположению индукции $G \in \Gamma_{N}(q-1) \cdot \Gamma_{N}(q-1)$. Тогда $G \in \Gamma_{N}(q) \cdot \Gamma_{N}(q)$ по утверждению 3.

2. Рассмотрим случай $G \in S_{N}$ и $|\Gamma(G)|=2 q-3$.

2а. Пусть в цикловой записи $G$ имеются циклы одинаковой длины

$$
G=\left(\alpha_{1}, \ldots, \alpha_{r}\right)\left(\beta_{1}, \ldots, \beta_{r}\right) G_{1}
$$


$\left|\Gamma\left(G_{1}\right)\right|=2(q-r)-3, \quad 2 \leq r<q-1$. Обозначим $\Omega^{\prime}=\{1, \ldots, N\} \backslash\left\{\alpha_{1}, \ldots, \alpha_{r}, \beta_{1}, \ldots, \beta_{r}\right\}$, $\left|\Omega^{\prime}\right|=N^{\prime}=N-2 r, \quad q^{\prime}=q-r<q, \quad G_{1} \in S\left(\Omega^{\prime}\right)$. При этом выполнено неравенство $q^{\prime} \leq \frac{N^{\prime}}{2}$.

Если при этом $q^{\prime} \geq 4$, то по предположению индукции существуют две подстановки $H_{1}, H_{2} \in S\left(\Omega^{\prime}\right), H_{1}, H_{2} \in \Gamma_{N^{\prime}}\left(q^{\prime}\right)$, для которых имеет место равенство $G_{1}=H_{1} \cdot H_{2}$. Тогда

$$
G=\left[\left(\alpha_{1}, \ldots, \alpha_{r}\right) \cdot H_{1}\right] \cdot\left[\left(\beta_{1}, \ldots, \beta_{r}\right) \cdot H_{2}\right],
$$

причем $\left(\alpha_{1}, \ldots, \alpha_{r}\right) \cdot H_{1},\left(\beta_{1}, \ldots, \beta_{r}\right) \cdot H_{2} \in \Gamma_{N}(q)$. Значит, $G \in \Gamma_{N}(q) \cdot \Gamma_{N}(q)$.

Если $q-r=3$, то $\left|\Gamma\left(G_{1}\right)\right|=3$, и подстановка $G_{1}$ является 3-циклом: $G_{1}=\left(\gamma_{1}, \gamma_{2}, \gamma_{3}\right)$. Тогда имеет место равенство

$$
G=\left[\left(\alpha_{1}, \ldots, \alpha_{q-3}\right) \cdot\left(\gamma_{1}, \gamma_{2}\right)\right] \cdot\left[\left(\beta_{1}, \ldots, \beta_{q-3}\right) \cdot\left(\gamma_{1}, \gamma_{3}\right)\right],
$$

из которого следует, что $G \in \Gamma_{N}(q-1) \cdot \Gamma_{N}(q-1)$. С учетом утверждения 3 получаем включение $G \in \Gamma_{N}(q) \cdot \Gamma_{N}(q)$.

Если $q-r=2$, то получаем противоречие $\left|\Gamma\left(G_{1}\right)\right|=1$.

2б. Пусть теперь в цикловой записи $G$ длины всех неединичных циклов различны. Поскольку число $2 q-3$ нечетно, то один из этих циклов имеет нечетную длину $r=2 t+1 \geq 3$ :

$$
G=\left(\alpha_{1}, \ldots, \alpha_{2 t+1}\right) G_{1}, \quad\left|\Gamma\left(G_{1}\right)\right|=2(q-t-1)-2 .
$$

Обозначим $\Omega^{\prime}=\{1, \ldots, N\} \backslash\left\{\alpha_{1}, \ldots, \alpha_{2 t+1}\right\},\left|\Omega^{\prime}\right|=N^{\prime}=N-2 t-1, q^{\prime}=q-t-1<q$, $G_{1} \in S\left(\Omega^{\prime}\right)$. При этом выполнено неравенство $q^{\prime} \leq \frac{N}{2}-t-1=\frac{N-2 t-2}{2}=$ $=\frac{N^{\prime}-1}{2}<\frac{N^{\prime}}{2}$.

Если при этом $q^{\prime} \geq 4$, то по предположению индукции существуют подстановки $H_{1}, H_{2} \in S\left(\Omega^{\prime}\right), H_{1}, H_{2} \in \Gamma_{N^{\prime}}\left(q^{\prime}\right)$, для которых выполняется равенство $G_{1}=H_{1} \cdot H_{2}$. Тогда

$$
G=\left[\left(\alpha_{1}, \ldots, \alpha_{t+1}\right) \cdot H_{1}\right] \cdot\left[\left(\alpha_{1}, \alpha_{t+2}, \ldots, \alpha_{2 t+1}\right) \cdot H_{2}\right],
$$

причем $\left(\alpha_{1}, \ldots, \alpha_{t+1}\right) \cdot H_{1},\left(\alpha_{1}, \alpha_{t+2}, \ldots, \alpha_{2 t+1}\right) \cdot H_{2} \in \Gamma_{N}(q-t-1+t+1)=\Gamma_{N}(q)$. 
Если $q-t-1=3$, то $\left|\Gamma\left(G_{1}\right)\right|=4$, и возможны два случая: либо $G_{1}$ является произведением двух независимых транспозиций, либо $G_{1}-4$-цикл.

В первом случае $G=\left(\alpha_{1}, \ldots, \alpha_{2 q-7}\right)\left(\beta_{1}, \beta_{2}\right)\left(\gamma_{1}, \gamma_{2}\right)$, откуда следует равенство $G=\left[\left(\alpha_{1}, \ldots, \alpha_{q-3}\right)\left(\beta_{1}, \beta_{2}\right)\right] \cdot\left[\left(\alpha_{1}, \alpha_{q-2}, \ldots, \alpha_{2 q-7}\right) \cdot\left(\gamma_{1}, \gamma_{2}\right)\right]$, означающее, что $G \in \Gamma_{N}(q-1) \cdot \Gamma_{N}(q-1)$. С учетом утверждения 3 получаем включение $G \in \Gamma_{N}(q) \cdot \Gamma_{N}(q)$.

Во втором случае при $q \geq 6$ подстановка $G$ сопряжена с подстановкой

$$
\tilde{G}=(1,2, \ldots, q-5, q, q+1, \ldots, 2 q-3)(q-4, q-3, q-2, q-1),
$$

которая представима в виде произведения

$$
\tilde{G}=(1,2, \ldots, q) \cdot[(q-4, q)(1, q+1, \ldots, 2 q-3)] \in \Gamma_{N}(q) \cdot \Gamma_{N}(q) .
$$

Значит, и в этом случае $G \in \Gamma_{N}(q) \cdot \Gamma_{N}(q)$.

Если $q-t-1=2$, то $\left|\Gamma\left(G_{1}\right)\right|=2$, и подстановка $G_{1}$ является транспозицией. При $q \geq 6$ подстановка $G$ сопряжена с подстановкой

$$
\tilde{G}=(1,2, \ldots, q-2, q, q-1, q+1, \ldots, 2 q-5)(2 q-4,2 q-3),
$$

которая представима в виде произведения

$$
\tilde{G}=(1,2, \ldots, q) \cdot[(2 q-4,2 q-3)(1, q-1, \ldots, 2 q-5)] \in \Gamma_{N}(q) \cdot \Gamma_{N}(q) .
$$

Значит, $G \in \Gamma_{N}(q) \cdot \Gamma_{N}(q)$.

Если $q-t-1=1, \quad$ то $\left|\Gamma\left(G_{1}\right)\right|=0$, т. е. $G=\left(\alpha_{1}, \ldots, \alpha_{2 q-3}\right)$. Тогда $G=$ $=\left(\alpha_{1}, \ldots, \alpha_{q-1}\right) \cdot\left(\alpha_{1}, \alpha_{q}, \ldots, \alpha_{2 q-3}\right) \in \Gamma_{N}(q-1) \cdot \Gamma_{N}(q-1)$. С учетом утверждения 3 и здесь получаем включение $G \in \Gamma_{N}(q) \cdot \Gamma_{N}(q)$.

3. Осталось рассмотреть случай $G \in S_{N}$ и $|\Gamma(G)|=2 q-2$.

3а. Пусть в цикловой записи $G$ имеются циклы одинаковой длины

$$
G=\left(\alpha_{1}, \ldots, \alpha_{r}\right)\left(\beta_{1}, \ldots, \beta_{r}\right) G_{1},\left|\Gamma\left(G_{1}\right)\right|=2(q-r)-2,2 \leq r \leq q-1 .
$$

Снова обозначим $\Omega^{\prime}=\{1, \ldots, N\} \backslash\left\{\alpha_{1}, \ldots, \alpha_{r}, \beta_{1}, \ldots, \beta_{r}\right\},\left|\Omega^{\prime}\right|=N^{\prime}=N-2 r$, $q^{\prime}=q-r<q, G_{1} \in S\left(\Omega^{\prime}\right)$. При этом выполнено неравенство $q^{\prime} \leq \frac{N^{\prime}}{2}$. 
Если при этом $q^{\prime} \geq 4$, то существуют две подстановки $H_{1}, H_{2} \in S\left(\Omega^{\prime}\right)$, $H_{1}, H_{2} \in \Gamma_{N}\left(q^{\prime}\right)$, для которых имеет место равенство $G_{1}=H_{1} \cdot H_{2}$. Тогда

$$
G=\left[\left(\alpha_{1}, \ldots, \alpha_{r}\right) \cdot H_{1}\right] \cdot\left[\left(\beta_{1}, \ldots, \beta_{r}\right) \cdot H_{2}\right],
$$

причем $\left(\alpha_{1}, \ldots, \alpha_{r}\right) \cdot H_{1},\left(\beta_{1}, \ldots, \beta_{r}\right) \cdot H_{2} \in \Gamma_{N}(q)$.

Если $q-r=3$, то $\left|\Gamma\left(G_{1}\right)\right|=4$, и возможны два случая: либо $G_{1}$ является произведением двух независимых транспозиций, либо $G_{1}-4$-цикл. В первом случае $G=\left(\alpha_{1}, \ldots, \alpha_{r}\right)\left(\beta_{1}, \ldots, \beta_{r}\right)\left(\gamma_{1}, \gamma_{2}\right)\left(\delta_{1}, \delta_{2}\right)$, очевидно, лежит в $\Gamma_{N}(r+2)$. $\cdot \Gamma_{N}(r+2)=\Gamma_{N}(q-1) \cdot \Gamma_{N}(q-1)$. С учетом утверждения 3 получаем включение $G \in \Gamma_{N}(q) \cdot \Gamma_{N}(q)$.

Во втором случае при $q \geq 7$ подстановка $G$ сопряжена с подстановкой

$$
\begin{aligned}
\tilde{G}=(1,2, \ldots, q-3)(q-2, q-1, q, 2 q-6,2 q-7, \ldots, q+1) \times \\
\\
\times(2 q-5,2 q-4,2 q-3,2 q-2),
\end{aligned}
$$

которая представима в виде произведения

$$
\begin{gathered}
\tilde{G}=(1,2, \ldots, q) \cdot[(1,2 q-6, \ldots, q+1, q-2)(2 q-5,2 q-4,2 q-3,2 q-2)] \in \\
\in \Gamma_{N}(q) \cdot \Gamma_{N}(q) .
\end{gathered}
$$

Значит, и в этом случае $G \in \Gamma_{N}(q) \cdot \Gamma_{N}(q)$.

Если $q-r=2$, то $\left|\Gamma\left(G_{1}\right)\right|=2$, и подстановка $G_{1}$ является транспозицией. Тогда подстановка $G$ сопряжена с подстановкой

$$
\tilde{G}=(1,2, \ldots, q-2)(q+1, \ldots, 2 q-2)(q-1, q),
$$

которая представима в виде произведения

$$
\tilde{G}=(1,2, \ldots, q) \cdot[(1, q-1)(q+1, \ldots, 2 q-2)] \in \Gamma_{N}(q) \cdot \Gamma_{N}(q) .
$$

Значит, и в этом случае $G \in \Gamma_{N}(q) \cdot \Gamma_{N}(q)$.

Если $q-r=1$, то $\left|\Gamma\left(G_{1}\right)\right|=0$, и подстановка $G_{1}$ является произведением двух циклов длины $q-1$. Значит, $G \in \Gamma_{N}(q-1) \cdot \Gamma_{N}(q-1) \subseteq \Gamma_{N}(q) \cdot \Gamma_{N}(q)$. 
3б. Осталось рассмотреть случай, когда $|\Gamma(G)|=2 q-2$, и все неединичные циклы подстановки $G$ имеют различную длину. Пусть $G$ является произведением $r$ циклов, длины которых равны $1<l_{1}<l_{2}<\ldots<l_{r}, \sum_{i=1}^{r} l_{i}=2 q-2$. Тогда $G$ сопряжена с подстановкой

$$
\tilde{G}=\left(1, \ldots, l_{1}\right)\left(l_{1}+1, \ldots, l_{1}+l_{2}\right) \ldots\left(l_{1}+\ldots+l_{r-1}+1, \ldots, 2 q-2\right) .
$$

Если $r=1$, то при $q \geq 5$ подстановка

$$
\begin{gathered}
(1,2, \ldots, q) \cdot[(1, q+1, \ldots, 2 q-4)(2,2 q-3,2 q-2)]= \\
=(1,2 q-3,2 q-2,2, \ldots, q, q+1, \ldots, 2 q-4),
\end{gathered}
$$

с одной стороны, лежит в $\Gamma_{N}(q) \cdot \Gamma_{N}(q)$, а с другой стороны, сопряжена с $\tilde{G}$. Значит, в рассматриваемом случае $G \in \Gamma_{N}(q) \cdot \Gamma_{N}(q)$.

Пусть теперь $r \geq 2$. Тогда существует такое $2 \leq s \leq r$, что

$$
\sum_{i=1}^{s-1} l_{i}<q, \quad \sum_{i=1}^{s} l_{i} \geq q
$$

Умножим подстановку $\tilde{G}$ слева на цикл длины $s-1\left(l_{1}, l_{1}+l_{2}, \ldots, l_{1}+\ldots+l_{s-1}\right)$, а справа - на цикл длины $r-s+1\left(l_{1}+\ldots+l_{s-1}+1, l_{1}+\ldots+l_{s}+1, \ldots, l_{1}+\ldots+l_{r-1}+1\right)$. Если $s-1$ или $r-s+1$ равны единице, то соответствующие циклы равны тождественной подстановке. В результате имеем подстановку

$$
\begin{gathered}
H=\left(l_{1}, l_{1}+l_{2}, \ldots, l_{1}+\ldots+l_{s-1}\right) \cdot \tilde{G} \cdot\left(l_{1}+\ldots+l_{s-1}+1, l_{1}+\ldots+l_{s}+1, \ldots, l_{1}+\ldots+l_{r-1}+1\right)= \\
=\left(1,2, \ldots, l_{1}, l_{1}+1, \ldots, l_{1}+l_{2}, \ldots, l_{1}+\ldots+l_{s-1}\right)\left(l_{1}+\ldots+l_{s-1}+1, \ldots, 2 q-2\right) .
\end{gathered}
$$

Обозначим $l_{1}+\ldots+l_{s-1}+1=m \leq q$. Можно заметить, что подстановка $H$ представима в виде произведения

$$
H=(1,2, \ldots, q) \cdot(m, 1, q+1, \ldots, 2 q-2) \in \Gamma_{N}(q) \cdot \Gamma_{N}(q) .
$$

Тогда

$$
\begin{gathered}
\tilde{G}=\left(l_{1}, l_{1}+l_{2}, \ldots, l_{1}+\ldots+l_{s-1}\right)^{-1} \cdot H \cdot\left(l_{1}+\ldots+l_{s-1}+1, l_{1}+\ldots+l_{s}+1, \ldots, l_{1}+\ldots+l_{r-1}+1\right)^{-1}= \\
=\left[\left(l_{1}, l_{1}+l_{2}, \ldots, l_{1}+\ldots+l_{s-1}\right)^{-1} \cdot(1,2, \ldots, q)\right] \times \\
\times\left[(m, 1, q+1, \ldots, 2 q-2) \cdot\left(l_{1}+\ldots+l_{s-1}+1, l_{1}+\ldots+l_{s}+1, \ldots, l_{1}+\ldots+l_{r-1}+1\right)^{-1}\right] .
\end{gathered}
$$


Рассмотрим подстановки

$$
\begin{gathered}
T_{1}=\left(l_{1}, l_{1}+l_{2}, \ldots, l_{1}+\ldots+l_{s-1}\right)^{-1} \cdot(1,2, \ldots, q), \\
T_{2}=(m, 1, q+1, \ldots, 2 q-2) \cdot\left(m, l_{1}+\ldots+l_{s}+1, \ldots, l_{1}+\ldots+l_{r-1}+1\right)^{-1} .
\end{gathered}
$$

Непосредственно убеждаемся, что

$$
\begin{gathered}
T_{1}=\left(l_{1}, l_{1}+l_{2}, \ldots, l_{1}+\ldots+l_{s-1}\right)^{-1} \cdot(1,2, \ldots, q)= \\
=\left(1,2, \ldots, l_{1}, l_{1}+\ldots+l_{s-1}+1, \ldots, q\right)\left(l_{1}+1, \ldots, l_{1}+l_{2}\right) \ldots\left(l_{1}+\ldots+l_{s-2}+1, \ldots, l_{1}+\ldots+l_{s-1}\right) .
\end{gathered}
$$

Значит, $T_{1} \in \Gamma_{N}(q)$. (Если $s=2$, то $T_{1}=(1,2, \ldots, q)$ также лежит в $\Gamma_{N}(q)$.)

Так как $\sum_{i=1}^{s} l_{i} \geq q$, то $\sum_{i=1}^{s} l_{i}+1 \geq q+1$.

Если $\sum_{i=1}^{s} l_{i}+1=q+1$, то

$$
\begin{gathered}
T_{2}=(m, 1, q+1, \ldots, 2 q-2) \cdot\left(m, q+1, \ldots, l_{1}+\ldots+l_{r-1}+1\right)^{-1}= \\
=(1, m)\left(q+1, \ldots, l_{1}+\ldots+l_{s+1}\right)\left(l_{1}+\ldots+l_{s+1}+1, \ldots, l_{1}+\ldots+l_{s+2}\right) \ldots \\
\ldots\left(l_{1}+\ldots+l_{r-1}+1, \ldots, 2 q-2\right) .
\end{gathered}
$$

При этом подстановка $T_{2}$ имеет

$$
2+l_{s+1}+\ldots+l_{r}=2 q-2-\left(l_{1}+\ldots+l_{s}\right)+2=2 q-2-q+2=q
$$

мобильных точек, т. е. $T_{2} \in \Gamma_{N}(q)$.

Пусть теперь $\sum_{i=1}^{s} l_{i}+1>q+1$. Тогда

$$
\begin{gathered}
T_{2}=(m, 1, q+1, \ldots, 2 q-2) \cdot\left(m, l_{1}+\ldots+l_{s}+1, \ldots, l_{1}+\ldots+l_{r-1}+1\right)^{-1}= \\
=\left(m, 1, q+1, \ldots, l_{1}+\ldots+l_{s}\right)\left(l_{1}+\ldots+l_{s}+1, \ldots, l_{1}+\ldots+l_{s+1}\right) \ldots\left(l_{1}+\ldots+l_{r-1}+1, \ldots, 2 q-2\right) .
\end{gathered}
$$

При этом подстановка $T_{2}$ имеет

$$
2+\left(l_{1}+\ldots+l_{s}-q\right)+l_{s+1}+\ldots+l_{r}=2 q-2-q+2=q
$$

мобильных точек, т. е. $T_{2} \in \Gamma_{N}(q)$. 
Итак, во всех возможных случаях доказано, что любая подстановка $G \in \Gamma_{N}(2 q-2)$ принадлежит множеству $\Gamma_{N}(q) \cdot \Gamma_{N}(q)$.

Теорема 4 доказана полностью.

Следствие. Если $N \geq 8, \quad 4 \leq q \leq \frac{N}{2}$ и $\mathcal{E}-$ тождественная подстановка, то

$$
\Gamma_{N}(q) \cdot \Gamma_{N}(q) \supseteq\left(\bigcup_{r=2}^{2 q-2} \Gamma_{N}(r)\right) \bigcup\{\varepsilon\} .
$$

ЗАМЕЧАНИЕ. Совершенно очевидно, что полученный результат не выполняется при $q \in\{2,3\}$. Действительно, множество $\Gamma_{N}(2)$ состоит из всех транспозиций, а множество $\Gamma_{N}(3)-$ из всех циклов длины 3 . Поэтому при $q \in\{2,3\}$ множество $\Gamma_{N}(q) \cdot \Gamma_{N}(q)$ состоит только из четных подстановок. Следовательно, в нем не содержится множество $\Gamma_{N}(2)$.

Далее рассмотрим, какие подстановки из множеств $\Gamma_{N}(2 q-1), \Gamma_{N}(2 q)$ принадлежат произведению $\Gamma_{N}(q) \cdot \Gamma_{N}(q)$. Пусть $G$ является произведением $r$ неединичных циклов, длины которых равны $m_{1}, m_{2}, \ldots, m_{r}$.

Утверждение 5. Пусть $N \geq 4, \quad 2 \leq q \leq \frac{N}{2}$, подстановка $G \in \Gamma_{N}(2 q)$ является произведением $r$ неединичных ииклов, длины которых равны $m_{1}, m_{2}, \ldots, m_{r}, \quad \sum_{i=1}^{r} m_{i}=2 q$. Подстановка $G$ представима в виде произведения двух подстановок из $\Gamma_{N}(q)$ в том и только в том случае, когда существует такое подмножество $\left\{i_{1}, \ldots, i_{k}\right\} \subseteq\{1, \ldots, r\}$, что $m_{i_{1}}+\ldots+m_{i_{k}}=q$.

Доказательство. Если для некоторых подстановок $H_{1}, H_{2} \in \Gamma_{N}(q)$ выполняется равенство $G=H_{1} \cdot H_{2}$, то $\Gamma\left(H_{1}\right) \cap \Gamma\left(H_{2}\right)=\varnothing$. Отсюда следует, что подстановки $H_{1}, H_{2}$ являются произведениями некоторых циклов из цикловой записи подстановки $G$. Пусть для подстановки $H_{1}$ длины этих циклов равны $m_{i_{1}}, \ldots, m_{i_{k}}$. Поскольку $H_{1} \in \Gamma_{N}(q)$, то $m_{i_{1}}+\ldots+m_{i_{k}}=q$.

Обратно, пусть искомое подмножество $\left\{i_{1}, \ldots, i_{k}\right\}$ существует. Обозначим $\left\{j_{1}, \ldots, i_{r-k}\right\} \subseteq\{1, \ldots, r\} \backslash\left\{i_{1}, \ldots, i_{k}\right\}$. Тогда $m_{j_{1}}+\ldots+m_{j_{r-k}}=2 q-q=q$. Построим подстановку $H_{1}$ как произведение циклов подстановки $G$, имеющих 
длины $m_{i_{1}}, \ldots, m_{i_{k}}$, а подстановку $H_{2}$ - как произведение циклов подстановки $G$, имеющих длины $m_{j_{1}}, \ldots, m_{j_{r-k}}$. Тогда $H_{1}, H_{2} \in \Gamma_{N}(q)$ и $G=H_{1} \cdot H_{2}$.

ЗАМЕЧАНИЕ. Из доказанного утверждения вытекает, что $\Gamma_{N}(2 q) \not \subset$ $\not \subset \Gamma_{N}(q) \cdot \Gamma_{N}(q)$. Действительно, $2 q$-циклы лежат в $\Gamma_{N}(2 q)$, однако они не удовлетворяют условию утверждения 5 .

Утверждение 6. Пусть $N \geq 4, \quad 2 \leq q \leq \frac{N}{2}$, подстановка $G \in \Gamma_{N}(2 q-1)$ является произведением $r$ неединичных илклов, длины которых равны $m_{1}, m_{2}, \ldots, m_{r}, \quad \sum_{i=1}^{r} m_{i}=2 q-1$. Подстановка $G$ представима в виде произведения двух подстановок из $\Gamma_{N}(q)$ в том и только в том случае, когда выполнено условие: существуют $i_{0} \in\{1, \ldots, r\}$ и такое подмножество $\left\{i_{1}, \ldots, i_{k}\right\} \subseteq$ $\subseteq\{1, \ldots, r\} \backslash\left\{i_{0}\right\}$, что $m_{i_{0}}>2 u \quad q-m_{i_{1}}+m_{i_{2}} \ldots+m_{i_{k}} \in\left\{2, \ldots, m_{i_{0}}-1\right\}$.

Доказательство. Если для некоторых подстановок $H_{1}, H_{2} \in \Gamma_{N}(q)$ выполняется равенство $G=H_{1} \cdot H_{2}$, то $\left|\Gamma\left(H_{1}\right) \cap \Gamma\left(H_{2}\right)\right|=1$. Пусть $\Gamma\left(H_{1}\right) \cap$ $\cap \Gamma\left(H_{2}\right)=\left\{\alpha_{1}\right\}$. Отсюда следует, что в подстановке $H_{1}$ существует цикл $\left(\alpha_{1}, \ldots, \alpha_{u}\right)$, в подстановке $H_{2}$ существует цикл $\left(\alpha_{1}, \beta_{2}, \ldots, \beta_{v}\right)$, а остальные циклы в $H_{1}, H_{2}$ являются циклами из цикловой записи подстановки $G$.

Пусть для подстановки $H_{1}$ длины этих циклов равны $m_{i_{1}}, \ldots, m_{i_{k}}$. Поскольку $H_{1} \in \Gamma_{N}(q)$, то $m_{i_{1}}+\ldots+m_{i_{k}}=q-u$. При вычислении произведения $H_{1} \cdot H_{2}$ получится цикл длины $u+v-1$

$$
\left(\alpha_{1}, \ldots, \alpha_{u}\right)\left(\alpha_{1}, \beta_{2}, \ldots, \beta_{v}\right)=\left(\alpha_{1}, \alpha_{2}, \ldots, \alpha_{u}, \beta_{2}, \ldots, \beta_{v}\right),
$$

причем этот цикл есть в цикловой записи подстановки $G$. Тогда выберем такое $i_{0} \in\{1, \ldots, r\}$, что $m_{i_{0}}=u+v-1$. Так как $u \geq 2, v \geq 2$, то $m_{i_{0}}>2$. Кроме того, $\left\{i_{1}, \ldots, i_{k}\right\} \subseteq\{1, \ldots, r\} \backslash\left\{i_{0}\right\}$ и $q-m_{i_{1}}+\ldots+m_{i_{k}}=u \in\left\{2, \ldots, m_{i_{0}}-1\right\}$.

Обратно, пусть искомое $i_{0} \in\{1, \ldots, r\}$ и подмножество $\left\{i_{1}, \ldots, i_{k}\right\}$ существуют. Обозначим $\left\{j_{1}, \ldots, i_{r-k-1}\right\} \subseteq\{1, \ldots, r\} \backslash\left\{i_{0}, i_{1}, \ldots, i_{k}\right\}$. Тогда

$$
\begin{gathered}
q-m_{i_{1}}+\ldots+m_{i_{k}}=u \quad \in\left\{2, \ldots, m_{i_{0}}-1\right\}, \\
m_{j_{1}}+\ldots+m_{j_{r-k-1}}=(2 q-1)-m_{i_{0}}-\left(m_{i_{1}}+\ldots+m_{i_{k}}\right)= \\
=(2 q-1)-m_{i_{0}}-q+u=q-\left(m_{i_{0}}+1-u\right) .
\end{gathered}
$$


Обозначим $v=m_{i_{0}}+1-u \in\left\{2, \ldots, m_{i_{0}}-1\right\}$. Тогда цикл $\left(\alpha_{1}, \ldots, \alpha_{m_{0_{0}}}\right)$ из цикловой записи подстановки $G$ можно представить в виде произведения двух циклов

$$
\left(\alpha_{1}, \ldots, \alpha_{m_{i 0}}\right)=\left(\alpha_{1}, \ldots, \alpha_{u}\right)\left(\alpha_{1}, \alpha_{u+1} \ldots, \alpha_{m_{i 0}}\right)
$$

длин $u$ и $v$ соответственно.

Построим подстановку $H_{1}$ как произведение циклов подстановки $G$, имеющих длины $m_{i_{1}}, \ldots, m_{i_{k}}$, и цикла $\left(\alpha_{1}, \ldots, \alpha_{u}\right)$. Подстановку $H_{2}$ построим как произведение циклов подстановки $G$, имеющих длины $m_{j_{1}}, \ldots, m_{j_{r-k-1}}$, и цикла $\left(\alpha_{1}, \alpha_{u+1} \ldots, \alpha_{m_{0}}\right)$. Тогда равенство $G=H_{1} \cdot H_{2}$ выполнено, подстановка $H_{1}$ имеет $m_{i_{1}}+\ldots+m_{i_{k}}+u=q$ мобильных точек, а подстановка $H_{2}$ имеет $m_{j_{1}}+\ldots+m_{j_{r-k-1}}+v=q$ мобильных точек.

ЗАМЕЧАНИЕ. Из доказанного утверждения вытекает, что $\Gamma_{N}(2 q-1) \not \subset$ $\not \subset \Gamma_{N}(q) \cdot \Gamma_{N}(q)$. Рассмотрим подстановки из $\Gamma_{N}(2 q-1)$, являющиеся произведением двух циклов длины $m_{1}=q$ и $m_{2}=q-1$. Покажем, что такие подстановки не могут удовлетворять условию из утверждения 6.

Если $i_{0}=1$, то в случае $\left\{i_{1}, \ldots, i_{k}\right\}=\{2\}$ имеем противоречие

$$
q-m_{i_{1}}+\ldots+m_{i_{k}}=q-(q-1)=1 \notin\left\{2, \ldots, m_{i_{0}}-1\right\} .
$$

В случае $\left\{i_{1}, \ldots, i_{k}\right\}=\varnothing$ снова имеем противоречие

$$
q-m_{i_{1}}+\ldots+m_{i_{k}}=q \notin\left\{2, \ldots, m_{i_{0}}-1\right\} .
$$

Если $i_{0}=2$, то в случае $\left\{i_{1}, \ldots, i_{k}\right\}=\{1\}$ имеем противоречие

$$
q-m_{i_{1}}+\ldots+m_{i_{k}}=q-q=0 \notin\left\{2, \ldots, m_{i_{0}}-1\right\} .
$$

В случае $\left\{i_{1}, \ldots, i_{k}\right\}=\varnothing$ снова имеем противоречие

$$
q-m_{i_{1}}+\ldots+m_{i_{k}}=q \notin\left\{2, \ldots, m_{i_{0}}-1\right\} .
$$

Итак, в теореме 4, утверждениях 5, 6 полностью описано строение множества $\Gamma_{N}(q) \cdot \Gamma_{N}(q)$ при $4 \leq q \leq \frac{N}{2}$. 


\section{Список литературы}

1. Bertram E. Even permutations as a product of two conjugate cycles // J. Combin. Theory (A). - 1972. - V. 12. No. 3. - P. 368-380.

2. Bertram E., Wei, $V$. K. Decomposing a permutation into two large cycles; an enumeration // SIAM J. Algebraic Discr. Meth. — 1980. - V. 1. No. 4. P. 450-461.

3. Boccara $G$. Cycles commi produit de deux permutations de classes donnes // Discrete Math. - 1982. - V. 38. No. 3. - P. 129-142.

4. Brenner J. L., Evans R. J. Even permutations as a product of two elements of order five // J. Combin. Theory (A). - 1987. — V. 45. No. 2. — P. 196-206.

5. Brenner $J$. L. Covering theorems for FINANSIGS VIII - almost all conjugacy classes in $A_{N}$ have exponent $\leq 4$ // J. Austral. Math. Soc. - 1987. V. 25. - P. 210-214.

6. Herzog M., Reid K. B. Representation of permutations as products of cycles of fixed length // J. Australian Math. Soc. (Ser. A). — 1976. — V. 22. No. 3. - P. 321-331.

7. Moran G. Reflection classes whose cubes cover the alternating group // J. Combin. Theory (A). - 1976. - V. 21. No. 1. — P. 1-19.

8. Moran G. Permutations as products of $k$ conjugate involutions // J. Combin. Theory (A). - 1975. - V. 19. No. 2. - P. 240-242.

9. Stanley R. P. Factorization of permutation into $n$-cycles // Discrete Math. 1981. - V. 37. No. 3. - P. 255-262.

10. Arad Z., Herzog M. eds. Product of conjugacy classes in groups // Lect. Notes Math. - Berlin: Springer-Verlag, 1985. - V. 1112. — 244 p.

11. Goupil A. On products of conjugacy classes of the symmetric group // Discrete Math. - 1990. - V. 79. No. 1. - P. 45-57.

12. Dvir Y. Covering properties of permutation groups // Lect. Notes Math. 1985. - V. 1112. - P. 197-221.

13. Тужилин М. Э. О порождении знакопеременной группы полурегулярными инволюциями // Обозр. прикл. и промышл. матем. - 2004. Т. 11. Вып. 4. - С. 938-939. 